Research Article

\title{
Improvement of the Voltage Profile and Loss Reduction in Distribution Network Using Moth Flame Algorithm: Wolaita Sodo, Ethiopia
}

\author{
S. Balakumar ${ }^{D},{ }^{1}$ Akililu Getahun, ${ }^{2}$ Samuel Kefale, ${ }^{2}$ and K. Ramash Kumar ${ }^{3}$ \\ ${ }^{1}$ Faculty of Electrical and Computer Engineering, Arba Minch University, Arba Minch-21, Ethiopia \\ ${ }^{2}$ Faculty of Electrical and Computer Engineering, Arba Minch University, Arba Minch-21, Ethiopia \\ ${ }^{3}$ Department of Electrical and Electronics Engineering, Dr.N.G.P. Institute of Technology, Coimbatore-48, Tamilnadu, India
}

Correspondence should be addressed to S. Balakumar; sbk25dec@gmail.com

Received 1 August 2021; Revised 18 November 2021; Accepted 19 November 2021; Published 1 December 2021

Academic Editor: Yang Li

Copyright $(2021 \mathrm{~S}$. Balakumar et al. This is an open access article distributed under the Creative Commons Attribution License, which permits unrestricted use, distribution, and reproduction in any medium, provided the original work is properly cited.

Voltage stability and line losses are inevitable issues even in modern power systems. There are several techniques that emerged to solve problems in the power system to provide quality and uninterrupted supply to customers. The algorithms used in this paper to determine the appropriate location and size of the Static Var Compensator (SVC) in the Distribution Network (DN) are Moth Flame Optimization (MFO) and Particle Swarm Optimization (PSO). The objective function is defined to minimize voltage deviation and power loss. The burning problem of voltage stability improvement current scenario is because of a rise in electricity demands in all sectors. Paramount duties of power engineers are to keep the system stable and maintain voltage magnitude constant even during peak hours. The results were checked with the aid of MATLAB on Wolaita Sodo radial distribution of 34 bus data networks. The potential use of SVC is key to solve distribution system power quality issues and estimating the advantage of the installation. The results obtained from the test system were compared with PSO results. This comparison was done to know the computational time of proposed techniques. The performance of the MFA based SVC was superior in distribution system and highlighted the importance of device.

\section{Introduction}

In modern times, distribution network structure is going through massive transformation. Due to the introduction of new technologies, distribution network structure is becoming more complex, and there is an ever-increasing demand for load. During voltage breakdown, voltage instability occurs, resulting in loss of voltage for a significant part of the system [1]. In order to increase voltage stability margin, FACTS controllers should be installed at the appropriate location to incorporate reactive power. FACTS devices comprise series, hybrid, and shunt devices. When dealing with a specific system voltage instability problem, use the correct component to restore system voltage stability [2]. Network reconfiguration (NR) is known as the process of modifying the network topology by reconfiguring the distribution network (DN).
Increasing the power flow tends to reduce the percentage power loss. Because of voltage profile improvements, smooth demand patterns, and improved network reliability, network reconfiguration offers major benefits [3]. The Particle Swarm Optimization (PSO) technique was used to achieve minimization active power losses and total function cost. This involves including the generation costs and the UPFC investment costs, which are deployed by installing the UPFC in the power system network to maximize benefits [4]. One novel optimization, inspired by nature, is presented in this study. The navigational strategy is employed by moths in the universe. The algorithm is known as the moth flame optimization (MFO) - a creative problem that presents engineering design and constrained optimization [5]. Many of the researchers have proved that MFO is one of the suitable algorithms for engineering problems. 
The authors like Yu et al. had solved power system issues and some of the engineering problems with MFO effectively. The MFO technique was successfully handled in the optimal placements of the DG [6].

There are keen researchers using MFA for the engineering problems like power engineering, computer engineering, and mechanical engineering. In this paper, the MFA algorithm was given quick results with less computational time than PSO. The moth flame optimization approach has also been used in medical and related sectors. The following are some of the studies of some academics. For the automatic detection of mitosis in breast cancer tissue pictures, Sayed and Hassanien [7] utilized neutrophils to optimize the moth flame population. Mingjing et al. [8] presented a new learning method for a nuclear limit learning machine using a chaotic moth flame optimization algorithm for medical diagnostics. In this work, a two-stage optimization strategy for optimal distributed generation (DG) planning with energy storage integration is provided.

The first stage uses the well-known Loss Sensitivity Factor (LSF) approach to determine the installation locations and initial capacity of DGs, and the second stage determines the optimal installation capacities of DGs to maximize investment benefits and system voltage stability and minimize line losses. The proposed two-stage optimization method outperforms current state-of-the-art DG planning approaches like NSGA-II, MOPSO, and MOHS, implying that our method achieves better outcomes across the range, including higher investment benefits, improved voltage stability, and lower line losses [9]. The Kenyan Power Network has seen significant increases in load demand in recent years. Although growing load demand has been partially met by additional transmission and generating capacity, due to economic, environmental, and geographic limits, the rate of growth has not kept pace with the rate of increase in load demand [10]. The goal of this research is to combine a microgrid system with a Static Synchronous Compensator (STATCOM) controller in order to ensure a higher power flow with a better voltage profile and less power loss. The microgrid and STATCOM controller connected to the grid are modelled using MATLAB/PSAT. The STATCOM is also controlled by Proportional Integral (PI) control and Fuzzy Logic Controller (FLC). By connecting to the main distribution system via a typical IEEE 30-bus connection, the usefulness of STATCOM with microgrid integration is assessed. Finally, it was discovered that STATCOM improves the voltage profile and reduces power loss by increasing the capacity of the distribution line [11].

The proposal's innovation is that it uses IPSO to optimize the model's parameters with synchro phasors, which enhances the prediction performance of the ELM-based TSP model. Finally, the correctness and validity of the provided approach are confirmed based on test results on both an IEEE 39-bus system and a large-scale real power system. Machine learning has shown to be effective in resolving online TSA issues. The existing PRTSA methodologies, on the other hand, are unable to fulfill the demands of big data administration and usage [12].
In this paper, various FACTS devices were tested as fault current limiters. Several FACTS devices are being explored for their performance, including the SSSC, STATCOM, and UPFC. SSSC has little impact on fault current and voltage regulation and instead focuses solely on reactive power flow. UPFC and STATCOM, on the other hand, can reduce fault current while also correcting voltage and regulating current. STATCOM and UPFC absorb reactive power from the system in such a way that fault currents are considerably reduced. UPFC outperforms STATCOM in terms of stability, transients, and voltage control. As a result, the crucial clearing time for the system will be extended [13].

\section{Statement of the Problem}

In this paper, Wolaita Sodo substation and its surroundings were taken as case study area. It consisted of several feeders, transformers, lines, and buses. The design of distribution system looks exactly radial which has 34 buses. During the data collection, researchers have noticed several problems associated with substation and around $300 \mathrm{~km}$ radius. The researchers aimed to registered current status of the substation by analysing the collected data. The values of voltage variations, power factor, harmonics, etc. had been measured by using MICROVIP3 PLUS. All the collected data were interpreted in the graphical structure. The overall data of the substation were collected and encapsulated in this work for future reference. The following graphs depicted various parameters values like voltage variation and harmonics. Let us view them one by one.

\subsection{Line Voltage Variation and Power Factor in Distribution} Network. This paper's study area was Wolaita Sodo distribution system which is located in the south part of Ethiopia. The geographical location is southern side of Addis Ababa, the capital city of Ethiopia; it has a latitude and longitude of $6^{\circ} 54^{\prime} \mathrm{N} 37^{\circ} 45^{\prime} \mathrm{E}$ with an elevation between 1600 and 2100 meters above sea level. For this study, voltage measurement is taken from Wolaita Sodo substation's all outgoing feeders control panel. Since the substation supplys $15 \mathrm{kV}$ to the distribution line, the voltage variation is occurring in the line. In Table 1 , the distribution line voltage $(\mathrm{kV})$ variation for peak time 8:00-21:00 is shown. This observed value leads researchers to focus on voltage profile of the distribution network.

In Figure 1, the voltage variation of Sodo line 2 from the substation control panel is shown. The data was measured in the intervals of one hour. There are drastic changes in the load around Wolaita substation due to residential and industrial enlargement. If observed the obtained graph Figure 1 it was clearly reflecting the variation in the voltage from morning 8 a.m to 8 p.m. the lowest voltage point registered in the graph on particular day was around $14.2 \mathrm{kV}$. The power factor is a vital issue in the distribution level. The low power factor may lead to line losses and cause deteriorate the performance of the system. This power factor issues can be sort out by external devices like FACTS family because it is local phenomena. In this analysis, researchers were tried to adapt the optimal device to improve voltage profile in 
Table 1: Distribution line voltage $(\mathrm{kV})$ variation for peak time 8:00-21:00.

\begin{tabular}{|c|c|c|c|c|c|c|}
\hline Time (s) & Gesuba line $1(\mathrm{kV})$ & Sodo line $2(\mathrm{kV})$ & Bodit line $4(\mathrm{kV})$ & Bilate line $5(\mathrm{kV})$ & $33 \mathrm{kV}$ Abala line $2(\mathrm{kV})$ & $33 \mathrm{kV}$ Bedesa line $3(\mathrm{kV})$ \\
\hline $8: 00$ & 14.8 & 14.6 & 14.8 & 14.8 & 32.7 & 32.8 \\
\hline $9: 00$ & 14.7 & 14.5 & 14.8 & 14.8 & 32.8 & 32.7 \\
\hline $10: 00$ & 15 & 14.7 & 14.7 & 14.7 & 32.8 & 32.7 \\
\hline $11: 00$ & 14.7 & 14.2 & 15 & 15 & 33 & 33 \\
\hline $12: 00$ & 15 & 14.68 & 15 & 15 & 33.1 & 33 \\
\hline $13: 00$ & 15 & 14.2 & 15 & 15 & 33 & 33 \\
\hline $14: 00$ & 15 & 14.65 & 15 & 15.1 & 32.8 & 32.7 \\
\hline $15: 00$ & 14.8 & 14.6 & 14.8 & 15 & 32.7 & 32.7 \\
\hline $16: 00$ & 15.1 & 14.7 & 15.1 & 14.8 & 32.8 & 32.8 \\
\hline $17: 00$ & 15 & 14.67 & 15 & 15.1 & 33 & 33.1 \\
\hline $18: 00$ & 15 & 14.76 & 15 & 15 & 33 & 33 \\
\hline $19: 00$ & 14.8 & 14.7 & 15 & 15 & 33 & 33 \\
\hline $20: 00$ & 15 & 14.5 & 14.9 & 14.9 & 32.9 & 33 \\
\hline
\end{tabular}

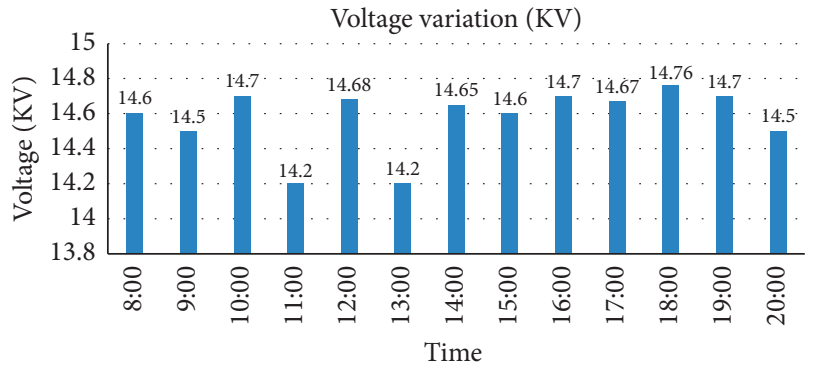

Figure 1: Voltage variation for Sodo line 2 from the substation control panel.

distribution system. The power factor for the distribution line of substation observed from the control panel is shown in Table 2.

In the distribution line, the voltage drop occurs due to long-distance coverage to achieve the utility's coverage. There is universal truth that power flow always flows from higher $\delta$ to lower $\delta$; likewise, reactive power flows from higher voltage to lower voltage-in general, sending end to receiving end-but this will be failed sometimes in case of reactive power due to uneven voltage profile at distribution system. Such common issues could be resolved with aid of FACTS devices. In this work, SVC plays a superior role in enhancing voltage throughout Wolaita Sodo distribution network. There are 34 buses dedicated to distributing power to the consumers. All the buses voltage must be near to knee point ( 0.95 to $1.05 \mathrm{P} . \mathrm{u})$. The moth flame algorithm was used for placing and sizing of the SVC in the network.

The main emphasis of the work was voltage profile improvement. The researchers were focused on and investigated reactive power support for the system and worked on maintaining constant voltage with the help of optimizationbased SVC. Figure 2 shows the photograph while taking measurements by using MICROVIP3 PLUS.

\section{Research Gap of the Work}

Voltage profile improvement and loss minimization were successfully obtained using MFA in the distribution network. Voltage deviation and line losses are indispensable problems even in modern power system. When the undesirable symptoms recurred, the power engineers diagnosed something different to tackle issues. Nowadays, maintaining the distribution system under normal condition is a big challenge due to all countries, particularly for developing countries. All researchers begin the quest for a reliable artificial intelligence method to settle the existing problems. In such a manner, in this work, searching for different and feasible algorithms has intensified. Finally, with deep analysis and literature review, MFA has been selected for optimization techniques. It was tested on Wolaita Sodo 34-bus system successfully and to show greatness of the work, load of the tested system had changed randomly to register changing of the location and size. The proposed algorithm could work on any kind of distribution system; depending on real and reactive power demands, it will optimize the place and size of the FACTS devices. Therefore, research gap of the work is clearly fulfilled and executed in the respective sections of the paper.

\section{Moth Flame Optimization}

Seyedali Mirjalili introduces the Moth-Flame Optimizer in 2015. MFO is an algorithm based upon population and represents the moths in the set of matrix:

$$
M=\left[\begin{array}{cccc}
m_{11} & m_{11} & \ldots & m_{1 d} \\
\vdots & \vdots & \vdots & \vdots \\
m_{21} & m_{22} & \ldots & m_{2 d} \\
\vdots & \vdots & \vdots & \vdots \\
m_{n 1} & m_{n 2} & \ldots & m_{n d}
\end{array}\right] .
$$

The numbers $n$ and $d$ are the number of moths (dimension) variables. In this paper, assume that the corresponding fitness values are stored in the following way for all moths [5]: where $n$ is the moth count,

$$
\mathrm{OM}=\left[\begin{array}{c}
\mathrm{om}_{1} \\
\vdots \\
\mathrm{om}_{2} \\
\mathrm{om}_{n}
\end{array}\right] .
$$


TABle 2: Power factor for the distribution lines of substation.

\begin{tabular}{|c|c|c|c|c|c|c|}
\hline Time (s) & Gesuba line 1 & Sodo line 2 & Bodit line 4 & Bilate line 5 & $33 \mathrm{kV}$ Abala line 2 & $33 \mathrm{kV}$ Bedesa line 3 \\
\hline $8: 00$ & 0.9 & 0.8 & 0.9 & 0.9 & 0.8 & 0.9 \\
\hline 9:00 & 0.8 & 0.87 & 0.8 & 0.8 & 0.9 & 0.8 \\
\hline $10: 00$ & 0.9 & 0.85 & 0.9 & 0.9 & 0.8 & 0.9 \\
\hline $11: 00$ & 0.9 & 0.88 & 0.8 & 0.8 & 0.8 & 0.8 \\
\hline $12: 00$ & 0.89 & 0.86 & 0.9 & 0.9 & 0.9 & 0.9 \\
\hline $13: 00$ & 0.9 & 0.87 & 0.9 & 0.9 & 0.9 & 0.9 \\
\hline $14: 00$ & 0.9 & 0.84 & 0.8 & 0.8 & 0.8 & 0.9 \\
\hline $15: 00$ & 0.9 & 0.8 & 0.84 & 0.84 & 0.84 & 0.8 \\
\hline $16: 00$ & 0.94 & 0.87 & 0.87 & 0.87 & 0.87 & 0.84 \\
\hline $17: 00$ & 0.9 & 0.89 & 0.96 & 0.96 & 0.96 & 0.87 \\
\hline $18: 00$ & 0.89 & 0.89 & 0.95 & 0.94 & 0.95 & 0.91 \\
\hline $19: 00$ & 0.9 & 0.8 & 0.9 & 0.8 & 0.94 & 0.96 \\
\hline $20: 00$ & 0.9 & 0.81 & 0.8 & 0.91 & 0.9 & 0.91 \\
\hline
\end{tabular}

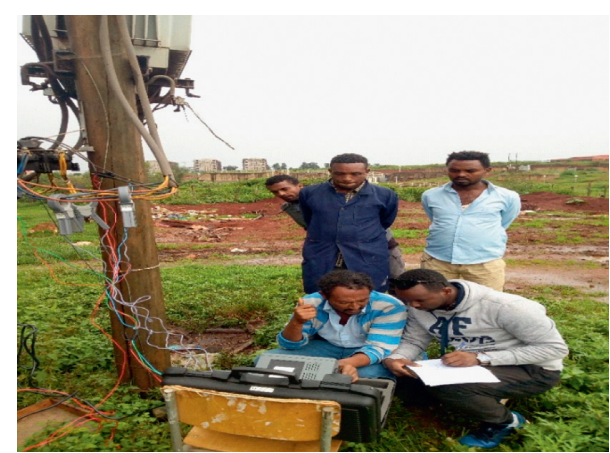

Figure 2: Photo during measurements.

Let us remember that the fitness value is the fitness return value (target) for each moth function. The fitness function and the output of the fitness function are passed to each moth position vector (first row in matrix $M$, for example) assigned as a fitness function to the corresponding moth (for example, OM Matrix OM). It is the moth set in a matrix. Moth flame optimization algorithm is conceived as transverse orientation by moth motion in nature. Moth flames could go right along the long distances of the night by keeping a fixed angle to their moon, but because of the artificial light they may move in a deadly spiral path. The moths are candidates for solutions in the proposed method, whereas the dependent variables or control variables are their position in space. The biggest distinction between the moths and the flames is that the moths are considered to be search agents migrating around the search area and that the flames portrayed by moth are the ultimate solution acquired. The flames are therefore the flags for moths; every moth moves around the flames and updates its position when a stronger decision is obtained, where $n$ is the moth number and $d$ the variable numbers (dimensions). Across all moths, researchers also assume that there is an array for the storing corresponding fitness values saved in array $(M)$. We understand that for every moth the value of fitness is the fitness return value (objective) function. Each fitness function is transferred to the position vector (e.g., first row in matrix $M$ ) of each moth and the fitness function output is delegated as the fitness function of the respective moth (for example, OMI in matrix and $\mathrm{OM}$ ). Flames are also the main components of the proposed algorithm. We consider a moth matrix-like matrix.

Researchers understand exactly that the $M$ and $F$ arrays dimensions are the same. For flames, authors also believe that the fitness values are saved in an array:

$$
\mathrm{OF}=\left[\begin{array}{c}
\mathrm{Of}_{1} \\
\mathrm{Of}_{2} \\
\vdots \\
\mathrm{Of}_{n}
\end{array}\right],
$$

where $n$ is the moth number. Moths and flames are both solutions. The difference is how each iteration is handled and updated. The moths are original search agents travelling around the search area, while flames are the best position to get moths up to now. Each moth is therefore looking for and updating a flame if a better solution is found. A moth has not ever lost its optimal answer with this technique.

\section{Moth Flame Algorithm for Placing and Sizing SVC}

\subsection{Step-by-Step Algorithm of $M F O$}

Step1: Read the information on the line and bus.

Step2: Compute power loss using a backward/forward sweeping load flow method, without an SVC connection. Deal with the power loss as the standard model.

Step3: Initialize moth population variables upper and lower limits, highest iterations and browse variable dimensions.

Step4: The random population (array) of the moth places continues to generate an initial array by equating appropriate with an array of the SVC sizes within defined by default top and bottom borders. Set the number of iteration $\left(k_{i}=\right.$ Zero).

Step5: Inspect whether the limitations are fulfilled or not in the moth positions.

Step6: If limitations have not been violated, compute the value of objective function (Complete loss of power) with load flow. 
Step7: Save as best flame the minimum value of the target function and the related to moth position as optimal flame position.

Step8: Using equation $(A)_{(i)}=\left(L_{i}\right.$ ebt.cos $\left.(2 \pi t)+B_{j}\right)$ Review the moth position to enable moths to converge to an optimal solution.

Step9: If exceed the maximum threshold of iteration, go to step5.

Step10: If not, update the $K=K+1$ iteration count and return to step5.

Step11: Break the best solution for the problem of optimization. The finest possible solution is to optimize SVC's location and size. The respective objective function value which embodies the lowest possible real power loss.

5.2. Flowchart of MFA. Figure 3 shows the exact function of the MFA. The coding of the algorithm was developed in the m-script of the MATLAB platform. In this section, crucial execution of the program was explained step by step. The results of the MFA had been compared with PSO. The system data could be read by the function $\left[d, V_{-}\right.$base $]=$data (). The maximum number of the bus and line could be taken by the command nbus $=\max (\max (d(:, 1)$. The results of load flow data were input to placing and sizing of SVC. In this optimization algorithm, maximum number of search agent is 30 . The maximum number of iterations is $100 . \mathrm{N}$ is denoted as number of search agent. The SVC rating is in $\mathrm{kVAR}$. The upper limit and lower limit were decided based on total reactive power demand of the system. The reactive power ranges from $-1 \mathrm{kVAR}$ to $+1 \mathrm{kVAR}$. The dimension of the problem was taken as 1 . Then initialize the positions of the moths using (4) and search randomly to fix best fitness.

$$
\text { Moth_pos }(:, 1)=\mathrm{SVC}_{\min }+\left(\mathrm{SVC}_{\max }-\mathrm{SVC}_{\min }\right) * \operatorname{rand}(N, 1) \text {. }
$$

The number of the flames in the work was decided by the following:

$$
\text { Flame_no }=\frac{\operatorname{round}(N-\operatorname{Iter} *(N-1))}{\text { Max_iteration }} .
$$

It is mandatory to check whether the moths are out of search space or not. If the moths are out of search space, it should be brought into search boundaries. The fitness of moths was calculated by MATLAB function:

$$
\text { Moth_fitness }(1, i)=\text { Obj_func }\left(\operatorname{Moth} \_\operatorname{pos}(i,:)\right) \text {. }
$$

The next steps after the fitness were sorting the first population of the moths based on best fitness. After the sorting of the flame, update the best position of the flames. The $8^{\text {th }}$ step in the algorithm gives the absolute value of the flame distance. The algorithm had converged after flame scored best value. The converged speed of the algorithm was faster than PSO in this work. In this paper, MFA dominated over the PSO for the placing and sizing the SVC in the test system.

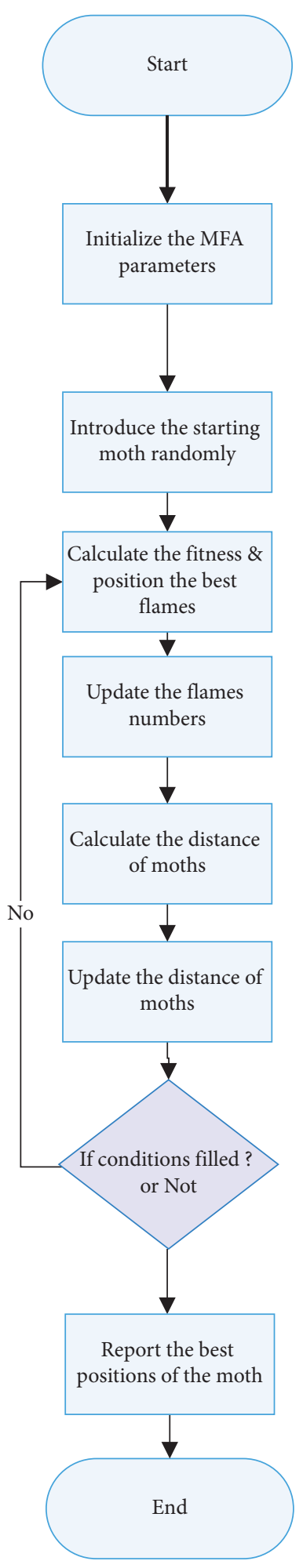

Figure 3: Flowchart of MFA.

\section{Results and Discussion}

6.1. Scenario 1. In this section, two different optimization techniques were used for sizing and placing the SVC in the Wolaita Sodo network. To ensure the suitable optimization techniques for the distributed network, different scenarios 
were formed and the effectiveness of the techniques was observed. The computational time of the algorithm was also taken as a crucial parameter to fix the superiority in the Wolaita Sodo distribution network. The suggested reasoning was tested on 34 radial bus systems and the results obtained were improved with MFA and PSO optimizations. The test system had a demand of 3.72 MW and 2.3 MVAR for real power and reactive capacity, respectively. The PSO technique is carried out on a radial 34-bus network, as per the standard steps. This MATLAB simulation program was developed and run on Intel Pentium dual core personal computer with 2 GB RAM. The final result obtained from the MATLAB software is shown in Table 3. It is evident that the PSO approach shows its advancement and benefits over the existing method in terms of line loss decrement and computational time.

Figure 4 clearly reiterates voltage deviation in the system while absence of SVC and voltage magnitude was restored in its optimal range with presence of SVC. In Figure 4, red colour line shows the results with SVC. Almost all 34 buses' voltage was maintained constant with reactive power support. The convergence line of PSO drawn on 34-bus system is also depicted in Figure 5. The iteration-1 starts objective function of 298.7986 and objective function is converged at iteration-100 value of 269.32 and elapsed time is 1.145873 seconds. Before SVC placement on optimal location, real power loss $(\mathrm{kW})$ is 369.57 and reactive power loss (kVAR) is 356.07. After optimal location and optimal sizing of SVC/, the real power and reactive power loss were reduced into $270.01 \mathrm{~kW}$ and $260.12 \mathrm{kVAR}$, respectively.

6.2. Scenario 2. The first scenario was tested successfully with PSO algorithm. In the second scenario, recent optimization technique, moth flame algorithm, was introduced for sizing and placing the SVC. This technique coding was developed in MATLAB and run on Intel Pentium dual core like PSO code. In Figure 6, results of voltage profile are constant in the entire distribution network. The range of the distribution system voltage started from 0.91 to $1.05 \mathrm{pu}$. The range in Figure 4 was accepted and matched with IEEE standard. This is one of the evidences that line losses would be minimized because of the constant voltage maintained in the system. The bus 7 was selected as weakest bus in the next for moment. The optimal size of the SVC was designed with MFA and placed at bus 7 for reactive power support. During the operation, bus 7 suffered more than the rest of the buses to maintain constant voltage at knee point.

The optimal size of the SVC had been decided value of $2300 \mathrm{kVAR}$. The active power loss in $\mathrm{kW}$ without SVC was $210.99 \mathrm{~kW}$ and with SVC $168.57 \mathrm{~kW}$. The percentage of the losses reduction in the system was $42.43 \mathrm{~kW}$. The reactive power losses without and with SVC were 143.13 and $126.26 \mathrm{kVAR}$, respectively. The percentage of reactive power loss observed was exactly $16.87 \mathrm{kVAR}$. The overall results of the work are showcased in Table 3. The comparisons were made between MFA and PSO in the all level performance. Figure 7 shows voltage profile of the 34 buses and all the bus voltage was maintained constant around $1 \mathrm{pu}$ after inclusion of SVC with MFA.
TABLE 3: Comparison results show location and rating of SVC and power loss reduction in 34-bus system.

\begin{tabular}{|c|c|c|c|}
\hline \multicolumn{2}{|c|}{ Techniques } & MFA & PSO \\
\hline \multicolumn{2}{|c|}{ Optimum placing of device } & Bus 7 & Bus 11 \\
\hline \multicolumn{2}{|l|}{ Optimal size of SVC } & 2300 & $1352 \mathrm{k}$ \\
\hline \multirow{2}{*}{$\begin{array}{l}\text { Active power loss } \\
(\mathrm{kW})\end{array}$} & $\begin{array}{l}\text { Without } \\
\text { SVC }\end{array}$ & 210.99 & $369.57 \mathrm{~kW}$ \\
\hline & With & 168.57 & $270.01 \mathrm{~kW}$ \\
\hline \multicolumn{2}{|c|}{$\%$ reduction in real power losses } & 42.42 & 27 \\
\hline \multirow{2}{*}{$\begin{array}{l}\text { Reactive power loss } \\
\text { (kVAR) }\end{array}$} & $\begin{array}{l}\text { Without } \\
\text { SVC }\end{array}$ & 143.13 & 356.07 \\
\hline & With SVC & 126 & $260.12 \mathrm{kVAR}$ \\
\hline \multicolumn{2}{|c|}{$\%$ reduction in reactive power losses } & $16.87 \mathrm{kVAR}$ & $27.4 \mathrm{kVAR}$ \\
\hline \multicolumn{2}{|c|}{ Computational time in sec } & $0.65 \mathrm{sec}$ & 1.145873 \\
\hline
\end{tabular}

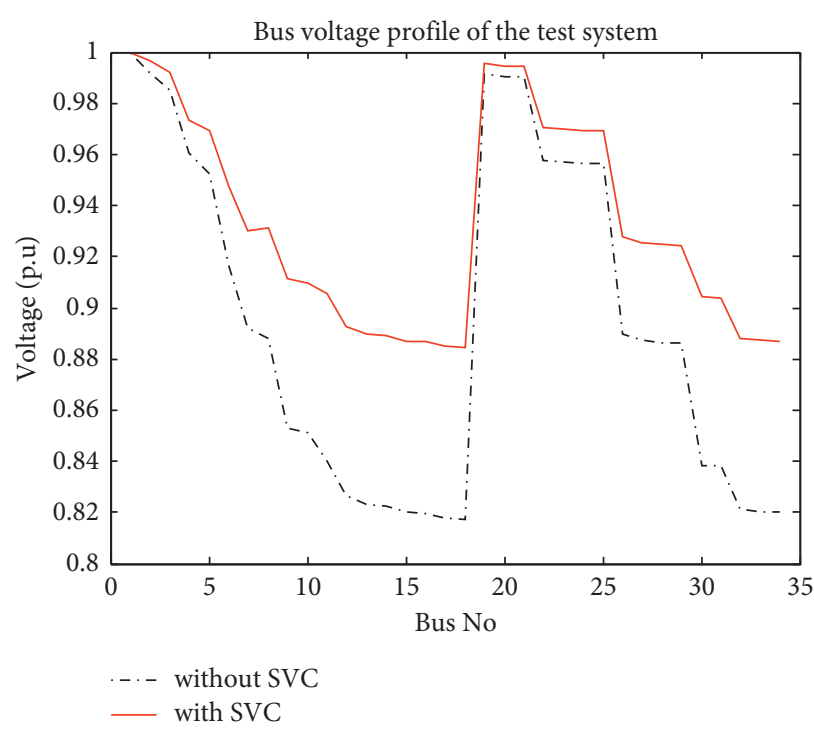

FIGURE 4: Voltage profile with and without SVC using PSO.

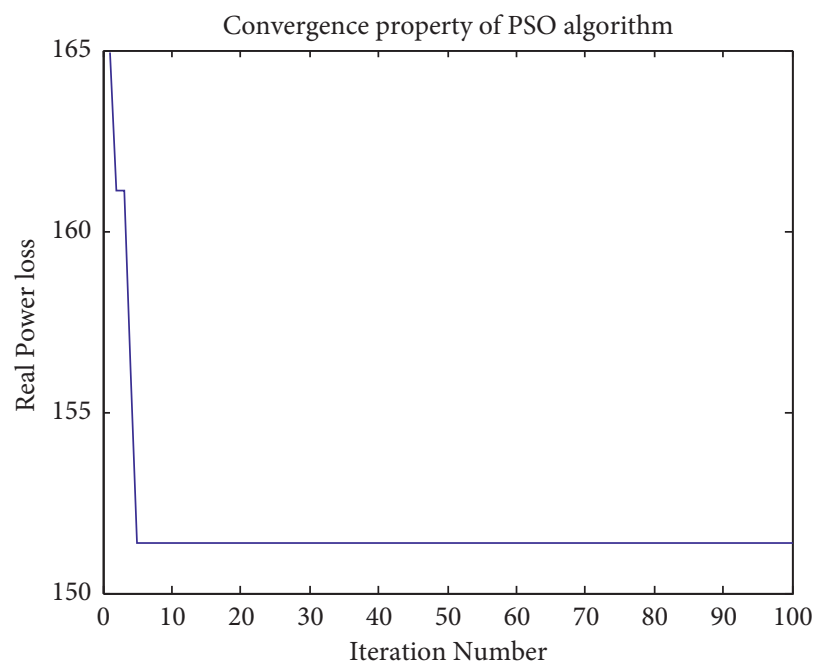

FIGURE 5: Convergence property of PSO algorithm.

The MFO technique had taken slight edge in terms of computational time and was given proper placing and sizing to fix the problem in the system. The power system has been 


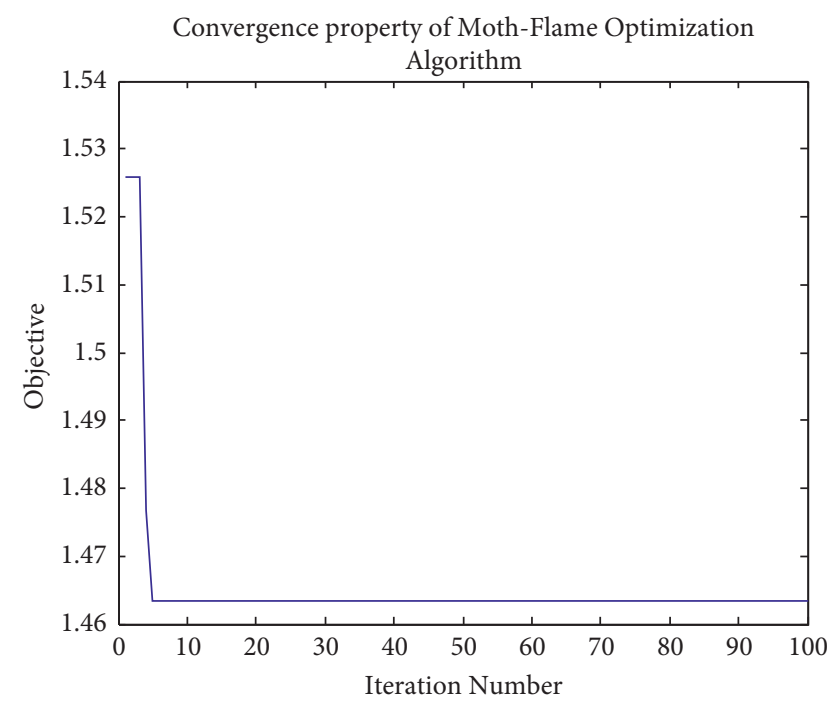

Figure 6: Convergence property of MFO.

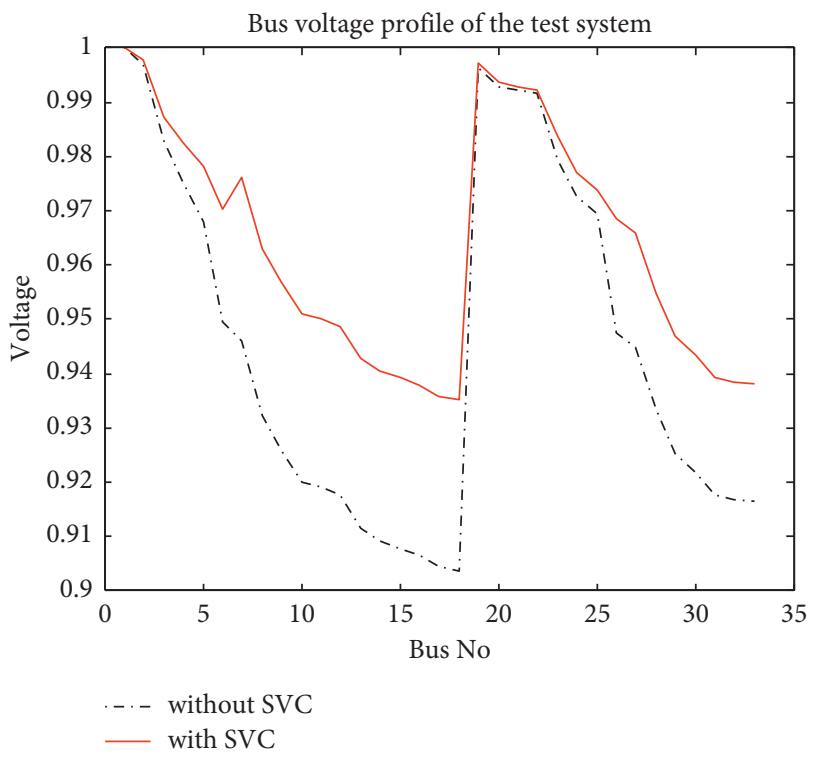

Figure 7: Voltage profile of 34-bus system with MFA.

more dynamical operation and uncertainty may occur any time. To prevent the faults, stability issues, compensation, and protection of the devices, the computerized monitoring system must act accordingly as fast as possible to take decision over the uncertainties. In that sense, MFA was shown its superiority in the case of improving voltage profile of the system. The big contribution of the work was helping to choose the rating of SVC during needed conditions. The operation of these proposed algorithms, MFA and PSO, would deliver exact sizing and placing of the SVC in the system. The detailed
TABle 4: Bus data for radial distribution system.

\begin{tabular}{|c|c|c|}
\hline \multirow{2}{*}{ Branch no. } & \multicolumn{2}{|c|}{ Nominal load at receiving bus } \\
\hline & Active load power $(\mathrm{kW})$ & Reactive power (kVAR) \\
\hline S/S 1 & 0 & 0 \\
\hline 2 & 24.4 & 14.5 \\
\hline 3 & 76.8 & 37.3 \\
\hline 4 & 74.9 & 46.2 \\
\hline 5 & 40.9 & 43.2 \\
\hline 6 & 35.9 & 13.5 \\
\hline 7 & 55.9 & 10 \\
\hline 8 & 8.95 & 3.39 \\
\hline 9 & 70.1 & 42.8 \\
\hline 10 & 78.5 & 20.8 \\
\hline 11 & 43.2 & 25.7 \\
\hline 12 & 43.61 & 23.6 \\
\hline 13 & 70.3 & 20.9 \\
\hline 14 & 78.2 & 9.56 \\
\hline 15 & 28.6 & 10.5 \\
\hline 16 & 14.8 & 7.23 \\
\hline 17 & 24.5 & 11.2 \\
\hline 18 & 25.3 & 8.62 \\
\hline 19 & 56.1 & 24.7 \\
\hline 20 & 36.1 & 14.3 \\
\hline 21 & 48.2 & 22.5 \\
\hline 22 & 20.5 & 15.6 \\
\hline 23 & 33.6 & 17.6 \\
\hline 24 & 30 & 15.5 \\
\hline 25 & 70.2 & 20.9 \\
\hline 26 & 55.9 & 10 \\
\hline 27 & 38.2 & 37.1 \\
\hline 28 & 43.61 & 23.6 \\
\hline 29 & 41.8 & 20.7 \\
\hline 30 & 140 & 85.3 \\
\hline 31 & 37.9 & 22.9 \\
\hline 32 & 25 & 28 \\
\hline 33 & 90.8 & 36.2 \\
\hline 34 & 21 & 10.2 \\
\hline
\end{tabular}

information of the collected and calculated data of Wolaita Sodo substation is recorded in Tables 4 to 7 . The data used in the work was measured from the case study substation. To implement the algorithm-based SVC in the system proper data from concern substation is mandatory. The MICROVIP3 PLUS device was used to measure harmonics and voltage variations in different places of the substations.

The collected 34-bus data is listed in Table 4. In Wolaita Sodo region, there are 34 distribution transformers under working conditions with different rating. All the transformer data are placed in Table 5. The line data of the system is catalogued in Table 6 and real and reactive power data of each bus during load flow execution is showcased in Table 7 . The overall data given in Tables $4-7$ could be useful for future enhancement of the realistic work in the same substation. 
TABle 5: Transformer data of the distribution system.

\begin{tabular}{|c|c|c|c|c|}
\hline Branch no. & Transformer (kVA) & No-load losses (W) & Full load losses (W) & $Z(\%)$ \\
\hline S/S 1 & 0 & 0 & 0 & \\
\hline 2 & 315 & 1100 & 3630 & 5.2 \\
\hline 3 & 100 & 280 & 2000 & 6 \\
\hline 4 & 50 & 350 & 1230 & 4 \\
\hline 5 & 315 & 820 & 4100 & 6 \\
\hline 6 & 200 & 890 & 2700 & 5.2 \\
\hline 7 & 200 & 900 & 3000 & 4.5 \\
\hline 8 & 100 & 280 & 2050 & 5 \\
\hline 9 & 315 & 950 & 3650 & 4 \\
\hline 10 & 100 & 520 & 1800 & 4 \\
\hline 11 & 630 & 456 & 2520 & 4.5 \\
\hline 12 & 200 & 100 & 2200 & 4 \\
\hline 13 & 630 & 1300 & 6500 & 4.5 \\
\hline 14 & 100 & 560 & 1820 & 4 \\
\hline 15 & 315 & 730 & 3650 & 4 \\
\hline 16 & 200 & 330 & 2860 & 5.5 \\
\hline 17 & 100 & 440 & 1760 & 4.5 \\
\hline 18 & 315 & 1050 & 4200 & 4 \\
\hline 19 & 100 & 500 & 1850 & 6 \\
\hline 20 & 100 & 440 & 1700 & 4 \\
\hline 21 & 200 & 120 & 2600 & 4 \\
\hline 22 & 200 & 600 & 2700 & 4.5 \\
\hline 23 & 100 & 600 & 1550 & 4 \\
\hline 24 & 25 & 110 & 350 & 4 \\
\hline 25 & 315 & 1300 & 4300 & 4 \\
\hline 26 & 50 & 220 & 1000 & 4 \\
\hline 27 & 200 & 600 & 2700 & 4.5 \\
\hline 28 & 100 & 320 & 1700 & 4.5 \\
\hline 29 & 315 & 1100 & 3780 & 5.3 \\
\hline 30 & 100 & 400 & 1550 & 4 \\
\hline 31 & 100 & 272 & 1505 & 4 \\
\hline 32 & 200 & 330 & 3700 & 4.13 \\
\hline 33 & 50 & 220 & 100 & 4 \\
\hline 34 & 25 & 210 & 695 & 4.5 \\
\hline
\end{tabular}

TABLE 6: Line impedance and admittance per unit on a distribution line.

\begin{tabular}{|c|c|c|c|c|c|c|c|}
\hline Branch no. & Sending end & Receiving end & Impedance $(Z$ pu $)$ & $R(\mathrm{pu})$ & $X(\mathrm{pu})$ & $|y|$ & $<Y$ \\
\hline 1 & 1 & 2 & $0.012196+0.05756 i$ & 0.0122 & 0.05756 & 16.9959 & 78 \\
\hline 2 & 2 & 3 & $0.02591+0.03344 i$ & 0.02591 & 0.03344 & 23.6389 & -52.2 \\
\hline 3 & 3 & 4 & $0.11651+0.07165 i$ & 0.11651 & 0.07165 & 7.3111 & -31.6 \\
\hline 4 & 4 & 5 & $0.03048+0.05504 i$ & 0.03048 & 0.05504 & 15.8942 & -61.02 \\
\hline 5 & 5 & 6 & $0.16952+0.17598 i$ & 0.16952 & 0.17598 & 4.09252 & -46.07 \\
\hline 6 & 6 & 7 & $0.15212+0.07592 i$ & 0.15212 & 0.07592 & 5.88191 & -26.52 \\
\hline 7 & 7 & 8 & $0.00204+0.07014 i$ & 0.00204 & 0.07014 & 14.2512 & -88.33 \\
\hline 8 & 8 & 9 & $0.26552+0.17672 i$ & 0.26552 & 0.17672 & 3.13526 & -33.64 \\
\hline 9 & 9 & 10 & $0.01855+0.00397 i$ & 0.01855 & 0.00397 & 52.7146 & -12 \\
\hline 10 & 10 & 11 & $0.09104+0.077876 i$ & 0.09104 & 0.07788 & 8.34698 & -40.5 \\
\hline 11 & 11 & 12 & $0.16519+0.0776 i$ & 0.16519 & 0.0776 & 5.47919 & -25.16 \\
\hline 12 & 12 & 13 & $0.02263+0.072987 i$ & 0.02263 & 0.07299 & 13.0865 & -72.77 \\
\hline 13 & 13 & 14 & $0.002089+0.01968 i$ & 0.00209 & 0.01968 & 50.5291 & -83.94 \\
\hline 14 & 14 & 15 & $0.091004+0.088996 i$ & 0.091 & 0.089 & 7.85625 & -44.36 \\
\hline 15 & 15 & 16 & $0.082178+0.02799 i$ & 0.08218 & 0.02799 & 11.5189 & -18.8 \\
\hline 16 & 16 & 17 & $0.169369+0.070098 i$ & 0.16937 & 0.0701 & 5.45548 & -22.48 \\
\hline 17 & 17 & 18 & $0.15014+0.085191 i$ & 0.15014 & 0.08519 & 5.79289 & -29.57 \\
\hline 18 & 2 & 19 & $0.04984+0.090347 i$ & 0.04984 & 0.09035 & 9.69158 & -61.11 \\
\hline 19 & 19 & 20 & $0.096827+0.08694 i$ & 0.09683 & 0.08694 & 7.68457 & -41.92 \\
\hline 20 & 20 & 21 & $0.09178+0.135156 i$ & 0.09178 & 0.13516 & 6.12097 & -55.82 \\
\hline
\end{tabular}


TABLE 6: Continued.

\begin{tabular}{lccccccc}
\hline Branch no. & Sending end & Receiving end & Impedance $(Z \mathrm{pu})$ & $R(\mathrm{pu})$ & $X(\mathrm{pu})$ & $|y|$ & $<Y$ \\
\hline 21 & 4 & 22 & $0.09178+0.136356 i$ & 0.09178 & 0.13636 & 6.08394 & -56.05 \\
22 & 22 & 23 & $0.0251+0.07067 i$ & 0.0251 & 0.07067 & 13.3342 & -70.45 \\
23 & 23 & 24 & $0.08867+0.10267 i$ & 0.08867 & 0.10267 & 7.3714 & -49.18 \\
24 & 24 & 25 & $0.02511+0.07067 i$ & 0.02511 & 0.07067 & 13.3336 & -70.44 \\
25 & 7 & 26 & $0.07015+0.07015 i$ & 0.07015 & 0.07015 & 10.0799 & -45 \\
26 & 26 & 27 & $0.173004+0.175156 i$ & 0.173 & 0.17516 & 4.06189 & -45.35 \\
27 & 27 & 28 & $0.08999+0.010676 i$ & 0.08999 & 0.01068 & 11.035 & -6.77 \\
28 & 28 & 29 & $0.071867+0.062764 i$ & 0.07187 & 0.06276 & 10.4804 & 41.13 \\
29 & 11 & 30 & $0.082142+0.09494 i$ & 0.08214 & 0.09494 & 7.96543 & -49.13 \\
32 & 30 & 31 & $0.0929067+0.08735 i$ & 0.09291 & 0.08735 & 7.84183 & -43.23 \\
31 & 14 & 32 & $0.08735+0.08589 i$ & 0.08735 & 0.08589 & 8.16303 & -44.51 \\
32 & 32 & 33 & $0.088996+0.081876 i$ & 0.089 & 0.08188 & 8.26927 & 42.61 \\
33 & 33 & 34 & $0.152498+0.090676 i$ & 0.1525 & 0.09068 & 5.63635 & -30.73 \\
\hline
\end{tabular}

TABLE 7: Each line real and reactive power in the system run in backward/forward MATLAB.

\begin{tabular}{|c|c|c|c|}
\hline \multicolumn{2}{|c|}{ Line } & \multicolumn{2}{|c|}{ Power at bus } \\
\hline From & To & $\mathrm{kW}$ & $\mathrm{kVAr}$ \\
\hline 1 & 2 & 423.598 & 1999.437 \\
\hline 2 & 3 & 1342.36 & 1732.404 \\
\hline 3 & 4 & 1725.787 & 1061.465 \\
\hline 4 & 5 & 843.7497 & 1523.622 \\
\hline 5 & 6 & 1188.448 & 1233.752 \\
\hline 6 & 7 & 1328.319 & 662.9177 \\
\hline 7 & 8 & 28.9178 & 996.4633 \\
\hline 8 & 9 & 962.906 & 640.9058 \\
\hline 9 & 10 & 853.0899 & 182.7174 \\
\hline 10 & 11 & 728.5451 & 623.197 \\
\hline 11 & 12 & 559.4409 & 262.6518 \\
\hline 12 & 13 & 170.2558 & 549.0849 \\
\hline 13 & 14 & 45.2561 & 426.3698 \\
\hline 14 & 15 & 111.1573 & 108.7036 \\
\hline 15 & 16 & 91.662 & 31.2216 \\
\hline 16 & 17 & 69.1257 & 28.6095 \\
\hline 17 & 18 & 22.9455 & 13.0193 \\
\hline 2 & 19 & 68.2395 & 123.7001 \\
\hline 19 & 20 & 70.2 & 63.0337 \\
\hline 20 & 21 & 25.5365 & 37.6061 \\
\hline 4 & 22 & 109.423 & 162.5714 \\
\hline 22 & 23 & 35.884 & 100.9834 \\
\hline 23 & 24 & 60.746 & 70.3374 \\
\hline 24 & 25 & 14.393 & 40.5043 \\
\hline 7 & 26 & 147.9189 & 193.5203 \\
\hline 26 & 27 & 112.8311 & 114.234 \\
\hline 27 & 28 & 91.468 & 10.8508 \\
\hline 28 & 29 & 34.0575 & 29.744 \\
\hline 11 & 30 & 130.5708 & 150.9173 \\
\hline 30 & 31 & 31.6605 & 29.7673 \\
\hline 14 & 32 & 107.5726 & 105.7773 \\
\hline 32 & 33 & 84.3736 & 77.6234 \\
\hline 33 & 34 & 20.0316 & 11.9109 \\
\hline
\end{tabular}

\section{Conclusion}

MFO algorithm is used in this article to find the optimum location, size, and SVC units to improve voltage profile and minimize the power loss in a Wolaita Sodo distribution of the 34-bus system. The findings indicated the efficiency of the algorithm, since the enforced MFO algorithm implies a lower power loss value than the literary results reported and PSO algorithm. The system's voltage profile is also improved immensely with SVCs being placed at relevant locations where more reactive power is required for voltage stable. The validity of the proposed system was noteworthy and results in different places in the articles were tabled. In this paper, effectiveness of the algorithm was compared with one of the popular algorithms PSO. Both algorithms hav effectively helped with maintaining constant voltage profile over long distances. The weakest zone of the network was bus 11 and bus 7 under different situations. The prolonged changes in the reactive power cause massive changes in the voltage which sometimes leads to brownout or blockout in the distribution ring. The moth flame optimization had been sized and placed the SVC in the optimal place for correcting the voltage deviations. The consequence of the constant voltage at all 34 buses in Wolaita Sodo leads to minimization in the line losses. The obtained results from the proposed system have proved that, under any uncertainty, conditions in Wolaita Sodo substation would be restored as soon as possible. The computational times of the algorithms were registered as $0.65 \mathrm{sec}$ for MFO and $1.14 \mathrm{sec}$ for PSO.

\section{Data Availability}

The data used to support the findings of this study are included within the article. The data were collected from Sodo Substation and given in Tables 4-7.

\section{Conflicts of Interest}

The authors declare that there are no conflicts of interest regarding the publication of this paper.

\section{References}

[1] M. A. Jirjees, D. A. Al-Nimma, S. Majid, and M. Al-Hafidh, "Voltage stability enhancement based on voltage stability indices using FACTS controllers," in Proceedings of the 2018 International Conference on Engineering Technologies and Their Applications (ICETA), Islamic University, AL Najaf, Iraq, 2018. 
[2] G. Deb, K. Chakra Borty, and S. Deb, "Voltage stability analysis using reactive power loading as indicator and its improvement by FACTS devices," in Proceedings of the 2016 IEEE 1st International Conference on Power Electronics, Intelligent Control and Energy Systems (ICPEICES), Delhi, India, 2016.

[3] G. Manikanta, A. Mani, and H. P. Singh, "Minimization of power losses in distribution system with variation in loads using adaptive quantum inspired evolutionary algorithm," in Proceedings of the 2018 4th International Conference on Computing Communication and Automation (ICCCA), IEEE, Greater Noida, India, 2018.

[4] A. R. Bhowmik and A. K. Chakraborty, "Placement of UPFC for minimizing active power loss and total cost function by PSO algorithm," in Proceedings of the 2013 International Conference on Advanced Electronics System, IEEE, Pilani, India, 2013.

[5] N. Jangir and I. N. Trivedi, "Moth-flame optimization algorithm for solving real challenging constrained engineering optimization problems," in Proceedings of the 2016 IEEE Students' Conference on Electrical, Electronics and Computer Science, IEEE, Bhopal, India, 2016.

[6] L. Yu, Z. Xinya, and L. Jingsen, "An improved moth-flame optimization algorithm for engineering problems," Symmetry, vol. 12, no. 8, 2020.

[7] G. I. Sayed and A. E. Hassanien, "Moth-flame swarm optimization with neutrosophic sets for automatic mitosis detection in breast cancer histology images," Applied Intelligence, vol. 47, no. 2, pp. 397-408, 2017.

[8] W. Mingjing, C. Huiling, Y. Bo et al., "Toward an optimal kernel extreme learning machine using a chaotic moth-flame optimization strategy with applications in medical diagnoses," Neurocomputing, vol. 267, pp. 69-84, 2017.

[9] L. Yang, B. Feng, G. Li, J. Qi, D. Zhao, and Y. Mu, "Optimal distributed generation planning in active distribution networks considering integration of energy storage," Applied Energy, vol. 210, 2017.

[10] O. Mogak, "Static voltage stability assessment of the Kenyan power network," Journal of Electrical and Computer Engineering, vol. 2021, Article ID 5079607, 16 pages, 2021.

[11] M. G. Yenealem, "Management of voltage profile and power loss minimization in a grid-connected microgrid system using fuzzy-based STATCOM controller," Journal of Electrical and Computer Engineering, vol. 2020, Article ID 2040139, 13 pages, 2020.

[12] Y. Zhang, T. Li, N. Guangyu, G. Li, and Y. Li, "Optimized extreme learning machine for power system transient stability prediction using synchro phasors," Mathematical Problems in Engineering, vol. 2015, Article ID 529724, 8 pages, 2015.

[13] M. Ibrahim, "Reducing fault current by using FACTS devices to improve electrical power flow," Mathematical Problems in Engineering, vol. 2021, Article ID 8116816, 9 pages, 2021. 